\title{
Reconstructions of the AlN(0001) surface
}

\author{
C. D. Lee, Y. Dong, and R. M. Feenstra \\ Department of Physics, Carnegie Mellon University, Pittsburgh, Pennsylvania 15213, USA \\ J. E. Northrup \\ Palo Alto Research Center, 3333 Coyote Hill Road, Palo Alto, California 94304, USA \\ J. Neugebauer \\ Fritz-Haber-Institut der Max-Planck-Gesellschaft, Faradayweg 4-6, D-14195 Berlin, Germany
}

(Received 26 June 2003; published 21 November 2003)

\begin{abstract}
Reconstructions of the AlN(0001) surface are studied. For moderately Al-rich surfaces, surface reconstructions with symmetry of $2 \sqrt{3} \times 2 \sqrt{3}-R 30^{\circ}$ and $5 \sqrt{3} \times 5 \sqrt{3}-R 30^{\circ}$ are found on the basis of scanning tunneling microscopy and low-energy electron diffraction observations. Such surfaces display a predominantly $2 \times 6$ pattern in reflection high-energy electron diffraction. Auger electron spectroscopy indicates an Al coverage for such surfaces of 2-3 monolayers. Based on this result and on first-principles total energy calculations it is argued that these reconstructions involve a laterally contracted $\mathrm{Al}$ adlayer structure similar to that previously proposed for $\mathrm{GaN}(0001)$. At higher $\mathrm{Al}$ coverages a thick, flat $\mathrm{Al}$ film is found to form on the surface. For Al-poor conditions, additional surface reconstructions with $\sqrt{3} \times \sqrt{3}-R 30^{\circ}$ and $2 \times 2$ periodicities are observed.

DOI: 10.1103/PhysRevB.68.205317

PACS number(s): 68.35.Bs, 68.55.Jk, 68.37.Ef, 61.14.Hg
\end{abstract}

\section{INTRODUCTION}

The $\mathrm{Al}_{x} \mathrm{Ga}_{1-{ }_{x}} \mathrm{~N}$ alloy system is employed in a variety of electronic and optoelectronic device structures including high-electron-mobility transistors, lasers, and light-emitting diodes. The energy band gap of this system may be varied over a range from 3.4 to $6.2 \mathrm{eV}$, with the upper part of the range becoming relevant in the push towards optoelectronic devices operating in the uv region. The growth of interfaces between alloys of differing group-III composition is needed in the fabrication of these devices, and control of the surface morphology is needed to ensure the formation of atomically abrupt interfaces. Knowledge of the atomic composition and structure of the growth surfaces of GaN and AlN is expected to facilitate the development of techniques, such as the use of surfactants, ${ }^{1}$ to improve the quality of the bulk material and of the interfaces between alloys of different composition.

Good progress has been made over the past six years in the understanding of surface structures of GaN. For both the (0001) and (0001) surfaces, i.e., Ga polarity and N polarity, respectively, a number of reconstructions have been observed as a function of surface stoichiometry. ${ }^{2}$ The detailed atomic arrangements for most of these reconstructions have been determined by a combination of scanning tunneling microscopy (STM) data and first-principles theoretical analysis. ${ }^{3-5}$ A unique aspect of the GaN surfaces, compared to other III-V semiconductors such as GaAs, is that they generally are terminated by excess numbers of the cation species, i.e., the $\mathrm{Ga}$ atoms. Termination by $\mathrm{N}$ atoms is energetically unfavorable, since those $\mathrm{N}$ atoms prefer to form $\mathrm{N}_{2}$ (with its strong $\mathrm{N}-\mathrm{N}$ bond) and thereby desorb from the surface.

In contrast to this progress in identifying surface structural arrangements on $\mathrm{GaN}$, there is only limited knowledge currently on the surface structures of AlN. Several studies have previously reported the symmetry of AIN surface reconstructions as seen by reflection high energy electron dif- fraction (RHEED) ${ }^{6-13}$ RHEED patterns with symmetries of $1 \times 1$ and $2 \times 2$ are commonly reported, a $2 \times 6$ pattern is also commonly seen, and a sequence consisting of $1 \times 3,3$ $\times 3$, and $6 \times 6$ is reported by several groups. (For RHEED on a surface with hexagonal symmetry, a $1 \times 3$ pattern usually is indicative of a $\sqrt{3} \times \sqrt{3}-R 30^{\circ}$ symmetry. ${ }^{14}$ ) Structural arrangements associated with these reconstructions have not been determined.

In this work we report on STM, RHEED, low-energy electron diffraction (LEED), and Auger electron spectroscopy (AES) measurements of reconstructions of the $\mathrm{AlN}(0001)$ surface. The reconstructions depend on the $\mathrm{Al}$ coverage, which can vary considerably since continued $\mathrm{Al}$ deposition at temperatures below about $750{ }^{\circ} \mathrm{C}$ is found to produce thick, flat films on the surface. (This result is in contrast to the case of $\mathrm{Ga}$ on $\mathrm{GaN}$ for which, at room temperature and above, any excess surface $\mathrm{Ga}$ above about 2 monolayers condenses into droplets.) For Al coverage of 2-3 monolayers $\left(\mathrm{ML}=1.19 \times 10^{15}\right.$ atoms $\left./ \mathrm{cm}^{2}\right)$ we observe a characteristic $2 \times 6$ RHEED pattern. From LEED it is found that this pattern originates from the $2 \sqrt{3} \times 2 \sqrt{3}-R 30^{\circ}$ surface unit cell. The $2 \sqrt{3} \times 2 \sqrt{3}-R 30^{\circ}$ unit cell, with a $\approx 10-\AA$ period, is observed in STM images together with a larger structure having $\mathrm{a} \approx 25-\AA$ period. This latter structure is identified as having a symmetry of approximately $5 \sqrt{3}$ $\times 5 \sqrt{3}-R 30^{\circ}$, and it is argued that it is derived from a surface lattice that is contracted and slightly rotated relative to the underlying AlN, such that the Al atom density in this surface lattice is close to that of bulk Al. On the basis of density functional pseudopotential calculations discussed later, it is argued that surfaces such as the $2 \sqrt{3} \times 2 \sqrt{3}-R 30^{\circ}$ and the $5 \sqrt{3} \times 5 \sqrt{3}-R 30^{\circ}$ probably incorporate a laterally contracted monolayer or bilayer structure containing a 4/3-ML Al layer contracted and rotated by $30^{\circ}$ such that it fits on the underlying $1 \times 1$ lattice. For Al films with thickness greater than a few ML we observe a characteristic $1 \times 1$ RHEED pattern 
but in which the diffraction streak spacing is about $6 \%$ greater than $1 \times 1$ AlN spacing, indicating a contraction of the lattice such that the $\mathrm{Al}$ atom density is close to that of bulk Al. For lower coverages of $\mathrm{Al}$ we observe additional reconstructions with symmetry $2 \times 2$ and $1 \times 3$.

\section{EXPERIMENT}

AlN films were grown by plasma-assisted molecular beam epitaxy (PAMBE) using a system previously described. $^{3-5}$ Most growths were performed on substrates consisting of $\approx 2-\mu \mathrm{m}$-thick GaN grown by metal-organic chemical vapor deposition (MOCVD) on sapphire. Onto this material we deposited, by PAMBE, $\approx 0.5-\mu$ m-thick $\mathrm{GaN}$ films. The starting GaN was known to have Ga polarity, and our GaN films clearly displayed in RHEED the pseudo-1 $\times 1$ reconstruction, thus confirming their Ga polarity. ${ }^{4}$ Depositing AlN directly on top of this GaN was found to yield rough surface morphology for AlN thicknesses greater than about $10 \mathrm{~nm}$, presumably because of the $-2.5 \%$ lattice mismatch of AlN relative to GaN. ${ }^{13}$ To alleviate this problem, a sequence containing $\approx 1$-nm-thick AlN layers followed by $\approx 2$-nm-thick GaN layers was deposited, with 5-10 periods, in an attempt to gradually grade the lattice constant to that of AlN. That sequence was doped with Si. An undoped AlN layer with a thickness of about $10 \mathrm{~nm}$ was then deposited on top. A somewhat flatter morphology was obtained in these cases compared to direct deposition of AlN on GaN. We assume that the final lattice constant is that of AlN, with an in-plane value of $3.11 \AA$, although some residual strain in the AlN layers could be present. In most cases a growth temperature of $750-800^{\circ} \mathrm{C}$ was used, although on a few occasions we have employed higher temperatures as discussed in Sec. III A. In a few instances AlN was directly deposited on $\mathrm{SiC}$, and a flat morphology was also achieved in that case (lattice mismatch of $+1.0 \%$ of AlN relative to $\mathrm{SiC}$ ). During and following growth the surface was characterized by RHEED. After the growth the samples were transferred under ultrahigh vacuum to an adjoining analysis chamber for study by STM and AES. STM was performed using a homebuilt instrument; calibration of the scanner is known from prior studies, but it does vary slightly depending on the scan speed and the length of the probe tip used. We thus use a $\pm 10 \%$ uncertainty (estimated from prior studies ${ }^{2-4}$ ) in the values quoted below.

AES was performed using a Perkin-Elmer 15-255G double-pass cylindrical mirror analyzer, at an incident electron energy of $3 \mathrm{keV}$. Our calculations of Auger line intensities make use of the Handbook of Auger Electron Spectroscopy values for the bulk sensitivity factors, ${ }^{15}$ which are converted to sensitivity factors for a layer of atoms using the known density of the standard materials and a phenomenological form for the electron escape depths. ${ }^{16}$ We then compute the expected intensity ratio of the $1396 K L L$ line of $\mathrm{Al}$ to the $379 \mathrm{eV} K L L$ line of N, for particular atomic arrangements of the AlN(0001) surface, and compare to experiment. As a check on the sensitivity factors, we use precisely the same analysis procedure on recently acquired data from $\mathrm{GaN}(0001)$ surfaces of known structure, ${ }^{3,4}$ using the 1070

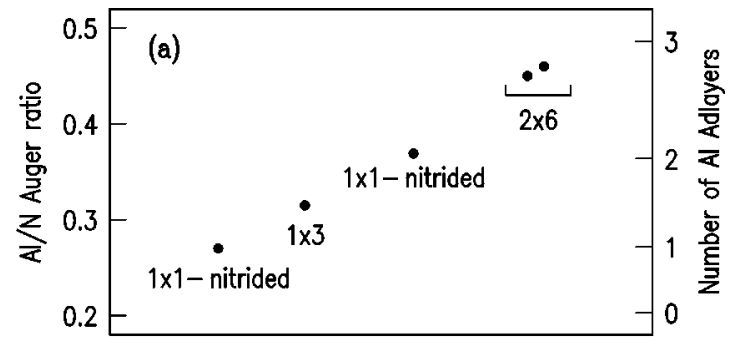

Al coverage

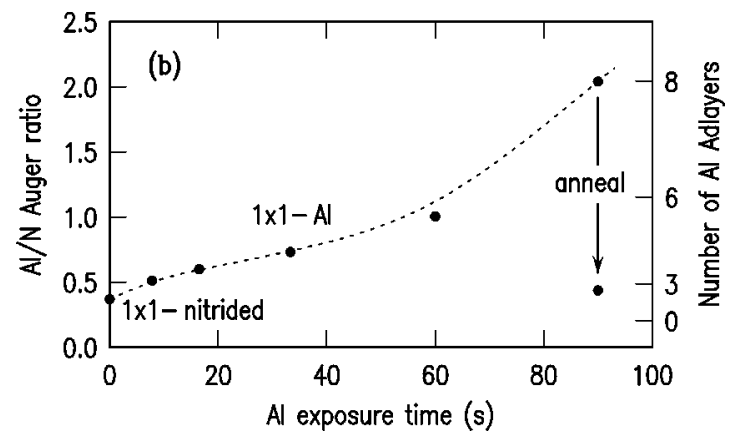

FIG. 1. Al/N intensity ratio measured by Auger electron spectroscopy in (a) low to moderate $\mathrm{Al}$ coverage range and (b) moderate to high Al coverage range. The right-hand vertical axes in (a) and (b) are based on model calculations and represent the number of $\mathrm{Al}$ monolayers residing on top of a Al-terminated AlN bilayer. The horizontal axis in (a) corresponds to a qualitative measure of $\mathrm{Al}$ coverage, based on preparation conditions for each reconstruction (labeled according to the observed RHEED patterns). The horizontal axis in (b) shows the exposure time to a fixed Al flux, with the sample held at $150{ }^{\circ} \mathrm{C}$. Following the sequence of depositions in (b), the sample was annealed at $750^{\circ} \mathrm{C}$ for $20 \mathrm{~min}$ and the Auger intensity ratio dropped to a value close to its starting value, as indicated by the arrow.

$L M M$ line of $\mathrm{Ga}$ and the $379 \mathrm{eV} K L L$ line of $\mathrm{N}$. We find computed ratios that are close, but not identical, to the measured ones. To achieve agreement between the two we find it necessary to increase the ratio of $\mathrm{Ga}$ to $\mathrm{N}$ sensitivity factors by $10 \%$. Given the similarity of the GaN and AlN structures, and of the core Auger emission between $\mathrm{Ga}$ and $\mathrm{Al}$, we therefore use this same $10 \%$ correction factor for the ratio of $\mathrm{Al}$ to $\mathrm{N}$ sensitivity factors in our present AlN analysis.

\section{RESULTS}

\section{A. Aluminum coverage ranges}

The Al content of our surfaces has been estimated from the AES measurements, as shown in Fig. 1. Figure 1(a) shows results for surfaces having less than about $3 \mathrm{ML}$ of $\mathrm{Al}$ coverage. Figure 1(b) shows results for higher Al coverages, obtained by successive depositions of $\mathrm{Al}$ on the $\mathrm{AlN}(0001)$ film, with the substrate held at about $150^{\circ} \mathrm{C}$. Specific surface structures, labeled according to their respective RHEED patterns, have been prepared using procedures described in Secs. III B-III D below. On the left-hand vertical axes in Fig. 1 we show the ratio of peak-to-peak intensities of the $\mathrm{Al}$ and $\mathrm{N}$ AES lines. On the right-hand axes we plot predictions of the $\mathrm{Al}$ surface coverage, i.e., the number of $\mathrm{Al}$ monolayers 
on top of an Al-terminated AlN bilayer, computed as described in Sec. II. The uncertainty in the predicted values of Fig. 1(a) is estimated to be about $\pm 0.5 \mathrm{ML}$, with perhaps greater uncertainty for the thicker Al coverages of Fig. 1(b). Some uncertainty in the experimental values also exists since the prepared surfaces are somewhat inhomogeneous, containing regions of greater and lesser $\mathrm{Al}$ content.

Figure 1(b) illustrates an important difference between metal deposition on $\mathrm{AlN}(0001)$ compared to $\mathrm{GaN}(0001)$, namely, that for AlN continued deposition of $\mathrm{Al}$ at room temperature or slightly above produces thick $\mathrm{Al}$ layers on the surface. As discussed in Sec. IIIC these thick Al layers are relatively flat, i.e., the $\mathrm{Al}$ does not form droplets as in the case of excess Ga on a GaN surface deposited at room temperature or above. ${ }^{4,17}$ When annealed at temperatures near $750{ }^{\circ} \mathrm{C}$ the thick $\mathrm{Al}$ films, however, form droplets, as discussed in Sec. III C (formation of Al droplets has previously been reported for Al-rich growth of AlN at temperatures ${ }^{11,18}$ of $775-850^{\circ} \mathrm{C}$ ).

\section{B. Moderate Al coverage}

Figure 2(a) shows a large-scale STM image, illustrating the general morphology of the AlN surfaces prepared for this study. For growth temperatures of $750-800^{\circ} \mathrm{C}$ a large number of growth spirals are present on the surface. These features form around threading dislocations with full or partial screw character, thus revealing a density of these defects of approximately $3 \times 10^{9} \mathrm{~cm}^{-2}$ for these films. This value is much greater than the threading screw dislocation density of $\approx 1 \times 10^{8} \mathrm{~cm}^{-2}$ found typically on the MOCVD-grown GaN films used as our substrates. In a few cases we have used higher growth temperatures of $850-880^{\circ} \mathrm{C}$ for the $\mathrm{AlN}$, and in that case the number of screw dislocations in the film is found to be significantly reduced, to about $3 \times 10^{8} \mathrm{~cm}^{-2}$.

During molecular beam epitaxy (MBE) growth, our AlN surfaces always display a $1 \times 1$ RHEED pattern. The growth is generally performed under Al-rich conditions, as seen from excess $\mathrm{Al}$ on the surface following growth as discussed below. If we terminate the growth by simultaneously turning off the $\mathrm{Al}$ and $\mathrm{N}$ sources and then cooling down the sample, the $1 \times 1$ (Al-rich) RHEED pattern seen during growth generally persists. Occasionally a $2 \times 6$ RHEED pattern can result directly from this type of growth termination, appearing at a temperature of $\approx 500^{\circ} \mathrm{C}$, and if not it can be obtained by performing a post-growth anneal at $800^{\circ} \mathrm{C}$ for about $10 \mathrm{~min}$. Longer annealing results in a $1 \times 1$ pattern. The surface displayed in Fig. 2 had been subjected to a postgrowth anneal that produced a $2 \times 6$ RHEED pattern. A hexagonal corrugation appears on the surface, seen in Fig. 1(b), with measured separation between corrugation maxima of $25 \pm 3 \AA$. This spacing is about 8 times that of the $1 \times 1$ surface lattice. Note that a number of distortions and stacking defects are evident in the surface corrugation of Fig. 1(b), some of which are marked in the image.

The long-period surface structure seen in Fig. 2(b) is the dominant one that we observe in STM images of surfaces that display a $2 \times 6$ RHEED pattern. However, a structure with shorter period has also been observed, as displayed in
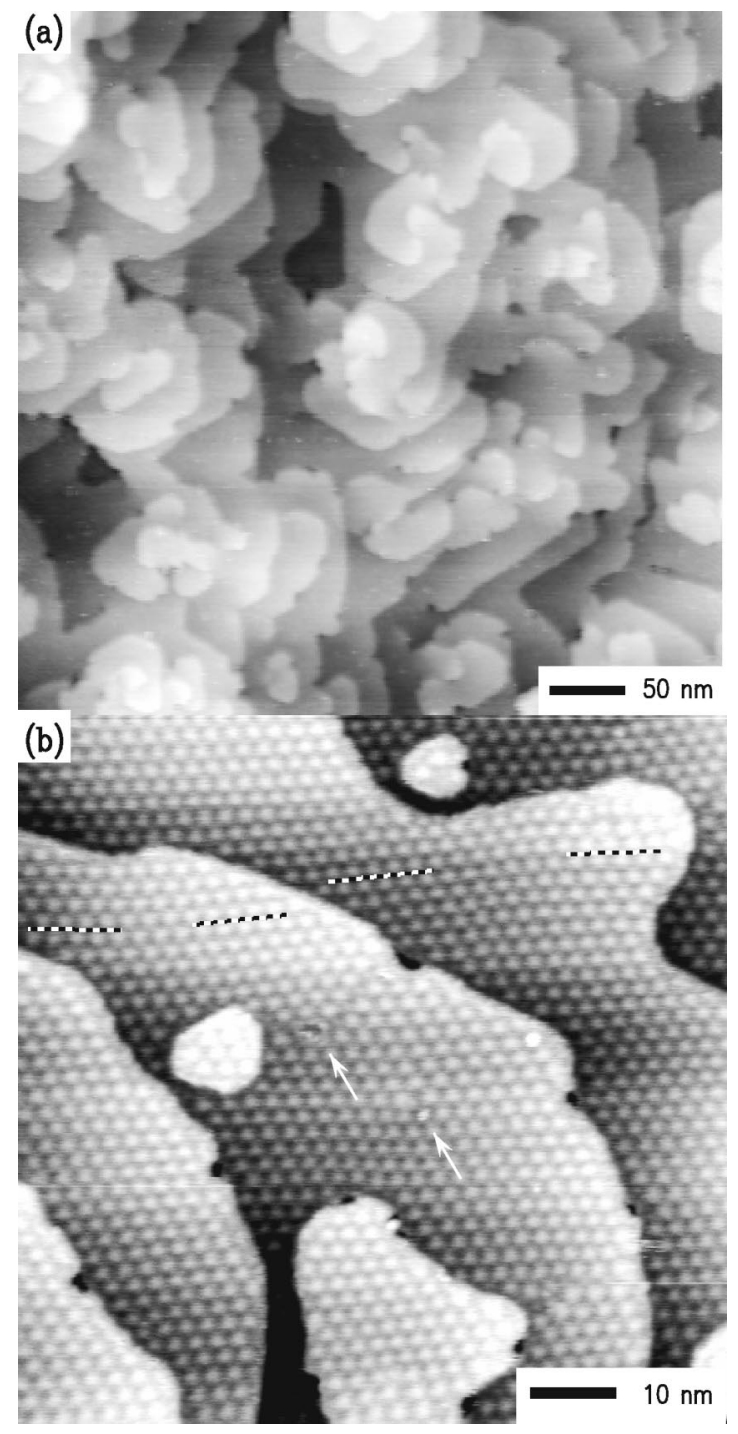

FIG. 2. STM images of Al-rich AlN(0001) surface. (a) Large scale image, acquired at $+1.0 \mathrm{~V}$ and displayed with gray scale range of $12 \AA$. (b) Magnified image, displaying the $5 \sqrt{3}$ $\times 5 \sqrt{3}-R 30^{\circ}$ reconstruction. Sample voltage is $+1.5 \mathrm{~V}$ and gray scale range is $3.5 \AA$. Some distortions in the hexagonal stacking sequence are marked by dashed lines, and the arrows mark surface dislocations.

Figs. 3 and 4. Figure 3 shows a terraced surface, most of which consists of the long-period structure similar to that of Fig. 2(b). Again, some distortions and defects in the stacking arrangement of this structure are evident. Additionally, in the terrace appearing at the lower left-hand corner of the image a new reconstruction can be seen. Again we find a hexagonal array of corrugation maxima, with separation between maxima in this case of $10 \pm 1 \AA$. We note that the orientations of the short-period and long-period corrugation are identical (i.e., they are not rotated by $30^{\circ}$ relative to each other). Figure 4 displays a surface region containing adjoining areas of the long-period and short-period structures, both with the same lattice constants as found from Figs. 2 and 3. The surface region shown in Fig. 4 again contains terraces, with the lower terrace $(A)$ consisting of the short-period 

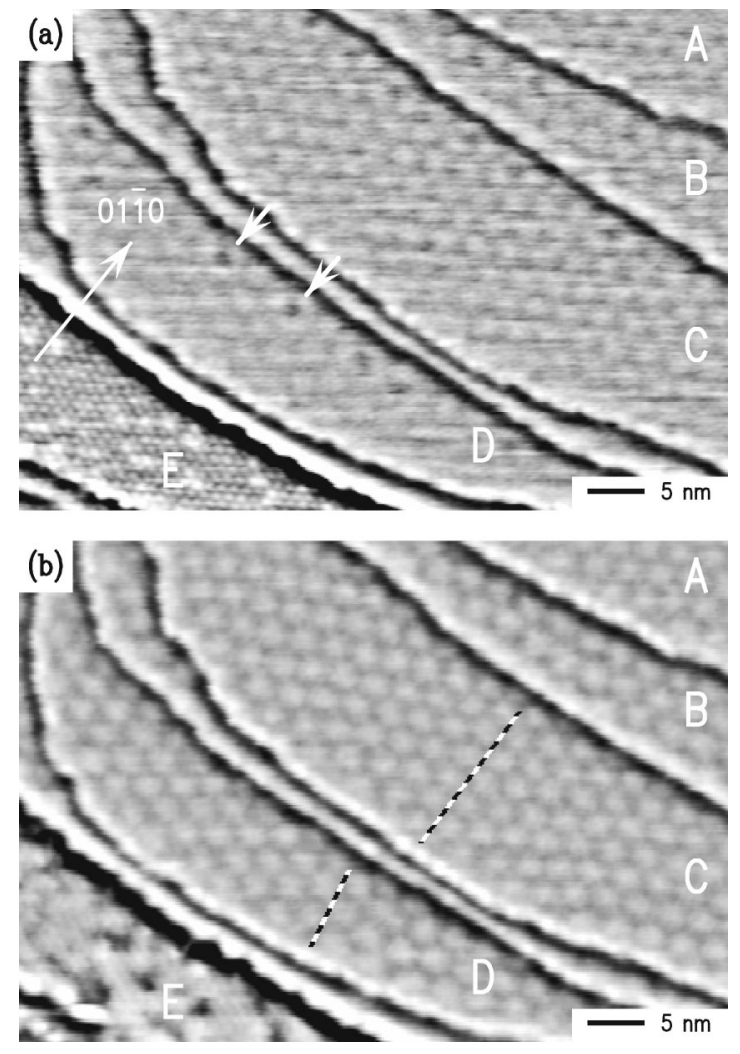

FIG. 3. STM images of a surface with multiple reconstructions, acquired with sample voltages of (a) $+3 \mathrm{~V}$ and $(\mathrm{b})+2 \mathrm{~V}$. The images are displayed using a background subtraction in which the background is formed by averaging the image over a window of $3 \times 3 \mathrm{~nm}^{2}$. The surface morphology actually consists primarily of five terraces, labeled $A-E$. A region of $2 \sqrt{3} \times 2 \sqrt{3}-R 30^{\circ}$ structure is seen in terrace $E$, and the remainder of the terraces consists of $5 \sqrt{3} \times 5 \sqrt{3}-R 30^{\circ}$ structure. Some distortions of the stacking sequence are evident, as marked by the dashed lines and arrows.

structure, and upper one $(C)$ being the long-period structure, and the intermediate terrace $(B)$ consisting of different region; $B_{1}$ and $B_{2}$ are long period, $B_{3}$ and $B_{4}$ are short period, and the structure of the small $B_{5}$ island is indeterminate. Using data such as those in Figs. 3 and 4, we can obtain a precise measure of the ratio of the periods of the two types of corrugation, and we find a value of $2.49 \pm 0.04$.

The symmetry of the observed reconstructions becomes clear once we inspect diffraction results. Typical $2 \times 6$ RHEED patterns are shown in Figs. 5(a) and 5(b). Sixfold streaks are clearly evident with the electron beam along $\langle 01 \overline{1} 0\rangle$, and weak additional streaks can sometimes be seen in the pattern. For the electron beam along $\langle 2 \overline{1} \overline{1} 0\rangle$, weak half-order streaks are apparent, and again additional weak streaks can sometime be seen. Furthermore, a relatively intense satellite fringe for both beam directions is always seen at wave vectors larger than those of the first-order streaks, as indicated by the white arrows in Figs. 5(a) and 5(b). We emphasize that the line intensities of our $2 \times 6$ RHEED patterns show subtle but distinct variations from surface to surface and also at different locations on the same surface, indicating that more than one type of reconstruction possesses
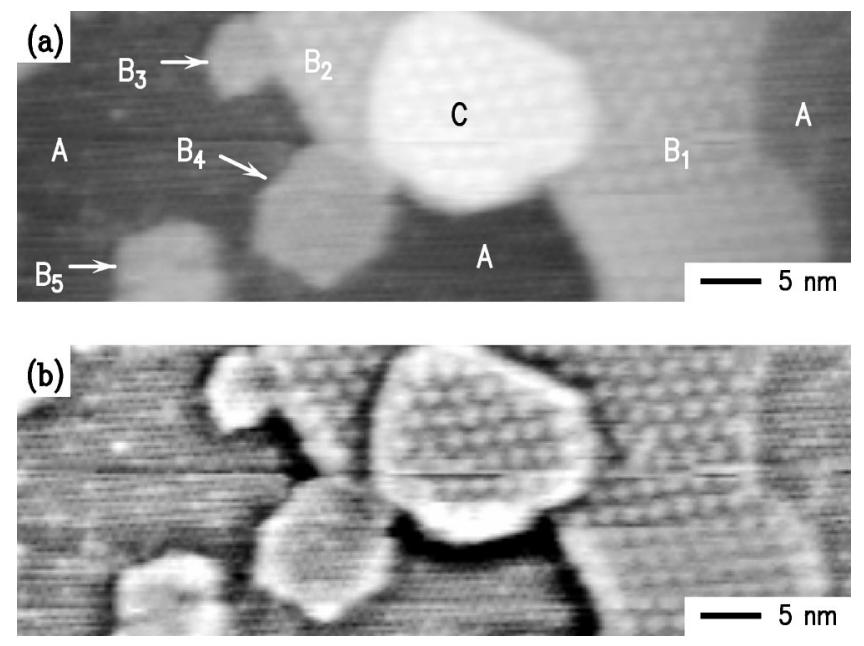

FIG. 4. STM image of a surface with multiple reconstructions, acquired at a sample voltage of $+2 \mathrm{~V}$. The same data are displayed in (a) and (b), but with different gray scale shading: (a) gray scale keyed to surface height, over a range of $0.9 \mathrm{~nm}$, and (b) gray scale computed using a background subtraction in which the background is formed by averaging the image over a window of $6 \times 6 \mathrm{~nm}^{2}$. The surface morphology consists of three terraces, labeled $A-C$ in panel (a). Terraces $A$ and $C$ consist of $2 \sqrt{3} \times 2 \sqrt{3}-R 30^{\circ}$ and $5 \sqrt{3}$ $\times 5 \sqrt{3}-R 30^{\circ}$ structure, respectively, and portions of terrace $B$ consist of one or the other structure.

this basic type of symmetry. Turning now to the LEED results, shown in Fig. 5(c), the six first-order spots are apparent, with spacing relative to the origin of $b=2 /(\sqrt{3} a)$ and located along $\langle 01 \overline{1} 0\rangle$ directions. At smaller wave vectors,
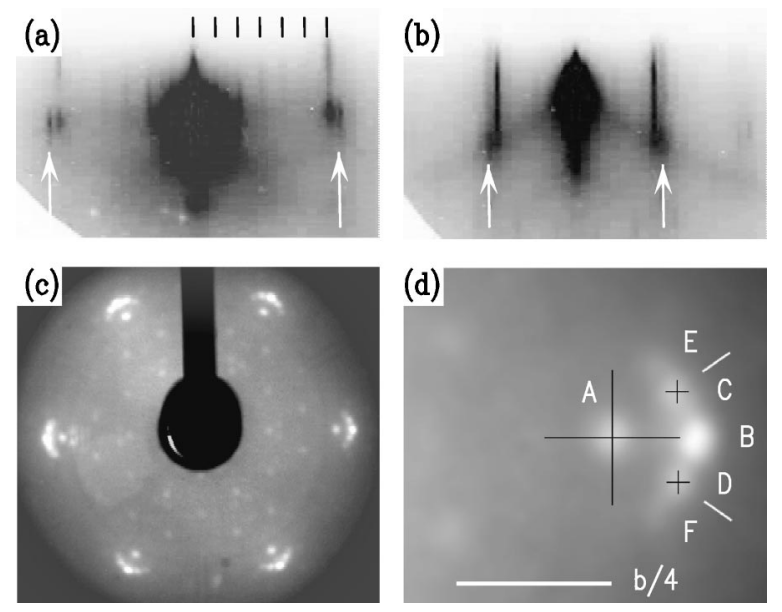

FIG. 5. Diffraction patterns from a moderately Al-rich AlN(0001) surface. (a) Reverse-contrast RHEED pattern with the electron beam along a $\langle 01 \overline{1} 0\rangle$ direction. Black tic marks indicate the location of $6 \times$ streaks. White arrows mark additional streaks seen at higher wave vectors. (b) Reverse-contrast RHEED pattern, with the electron beam along a $\langle 2 \overline{1} \overline{1} 0\rangle$ direction. White arrows mark the location of streaks occurring at high wave vector. (c) LEED pattern, acquired at $80 \mathrm{eV}$ electron energy. The leftmost firstorder peak is slightly distorted due to a stray light reflection in the optical system used to acquire the image. (d) Expanded view of first-order reflection and satellite peaks. 
additional spots with $b /(2 \sqrt{3})$ spacing located along both $\langle 2 \overline{1} \overline{1} 0\rangle$ and $\langle 01 \overline{1} 0\rangle$ directions are seen. From this arrangement it is clear that an underlying $2 \sqrt{3} \times 2 \sqrt{3}-R 30^{\circ}$ symmetry is present in the pattern. We conclude that the basic symmetry of the surface reconstructions is rotated by $30^{\circ}$ relative to the underlying $1 \times 1$ lattice. With this observation, the symmetries of the structures observed in STM is clear. For the observed $10 \pm 1 \AA$ spacing, whose length would be consistent with either $3 \times 3$ or $2 \sqrt{3} \times 2 \sqrt{3}-R 30^{\circ}$, we can identify the latter as being correct based on LEED. It is also important to note that a $2 \sqrt{3} \times 2 \sqrt{3}-R 30^{\circ}$ surface periodicity will produce a $2 \times 6$ pattern in RHEED (in the same sense as the well-known result that a $\sqrt{3} \times \sqrt{3}-R 30^{\circ}$ periodicity produces a $1 \times 3$ RHEED pattern ${ }^{14}$ ). For the long-period structure, with period 2.49 \pm 0.04 times larger than the short-period one and the same orientation, we identify it as $5 \sqrt{3}$ $\times 5 \sqrt{3}-R 30^{\circ}$.

Additional splittings of the LEED spots, particularly of the first-order spots, is apparent in Fig. 5(c) and are shown in an expanded view in Fig. 5(d). The first-order LEED spot in that image is labeled by $A$. Surrounding this first-order spot are the satellite spots labeled $B-F$, oriented at approximately $30^{\circ}$ intervals around a ring of radius $(0.125$ $\pm 0.007) b$. The radius of this ring agrees well with the observed spacing of the satellite fringes in the RHEED pattern of Fig. 5(b) (which corresponds to a cut through the firstorder spots of the LEED pattern), which have a spacing $(13 \pm 1) \%$ larger than the first-order fringe spacing. Also, the inverse of this radius, $21.5 \pm 1.2 \mathrm{~nm}$, is in agreement with the spacing of the corrugation lines seen in the STM images, $(\sqrt{3} / 2)(25 \pm 3) \mathrm{nm}=22 \pm 3 \mathrm{~nm}$.

Some additional features of the LEED pattern require more detailed consideration. Regarding the orientation of the satellite spots, the $C$ and $D$ spots are located at approximately $\pm 30^{\circ}$ relative to $\langle 01 \overline{1} 0\rangle$ around a circle centered at $A$. These locations are consistent with the rotated sense of the STM images relative to the underlying $1 \times 1$ lattice. However, the presence of the bright spot $B$ and the weak spots $E$ and $F$ are not consistent with this type of rotated symmetry. Moreover, the angular separation between $C$ and $D$ relative to $A$ is actually found to be somewhat larger than $60^{\circ}$, with a measured value of $69 \pm 3^{\circ}$. To understand these aspects of the data, we refer back to the STM images of Figs. 2 and 3. As seen there, considerable disorder in the orientation of the corrugation rows is seen, with angular deviations in the row directions of approximately $\pm 4^{\circ}$. The surface appears to be composed of many small domains, which vary in relative angular orientation by approximately this amount. Thus, we propose that the hexagonal network actually has two orientations, one specified by the wave vector $\vec{k}_{C}$ and the other by $\vec{k}_{D}$. The magnitude of $\vec{k}_{C}$ and $\vec{k}_{D}$ is measured to be $(1.102 \pm 0.006) b$ and their angular separation from a $\langle 01 \overline{1} 0\rangle$ direction is $3.9^{\circ} \pm 0.3^{\circ}$. Each of these wave vectors will produce a single, slightly rotated, hexagonal domain, and indeed a close inspection of Fig. 2 suggests that a collection of such domains is not inconsistent with the STM data. Now, to account for the bright spot $B$, we propose that disorder in the

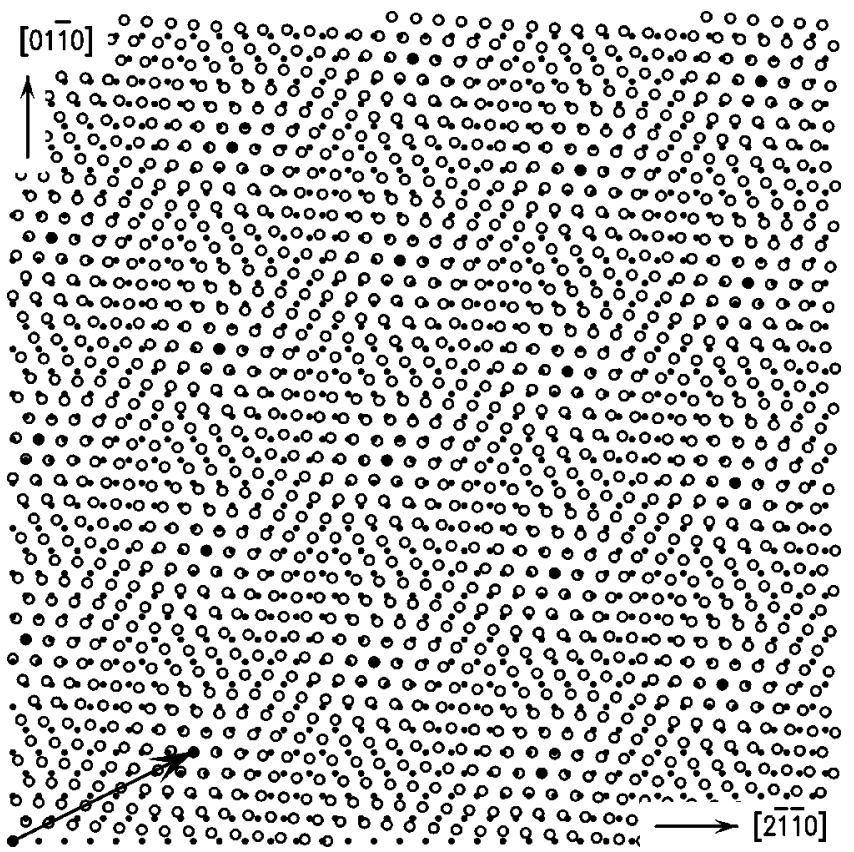

FIG. 6. Illustration of coincidence between two hexagonal lattices, shown by closed and open dots, respectively. The second lattice is contracted by 0.90185 and rotated by $3.6705^{\circ}$ relative to the first. A coincidence vector of the two lattices is shown. The resulting coincidence lattice has hexagonal (rotated) symmetry.

orientation of the two domains produces a coherent set of corrugation rows oriented at their average angular orientation. This proposal may seem somewhat surprising at first, since disorder usually produces a spot broadening and not a new, distinct spot. However, a suitable alternating series of corrugation rows, with relative orientation given by $\vec{k}_{C}, \vec{k}_{D}$, $\vec{k}_{C}$, etc., would lead to a well-defined spot at the location given by $B$. The STM images do indeed imply the presence of a well-defined long-range average spacing of corrugation rows, which is thus consistent with the presence of the $B$ spot.

To further investigate the formation of the $5 \sqrt{3}$ $\times 5 \sqrt{3}-R 30^{\circ}$ structure, and in particular its $\pm 3.9^{\circ}$ angular variants, we show in Fig. 6 a specific atomic model for such a structure. This model is obtained simply by considering the coincidence obtained when one overlays two lattices with slightly different lattice constants on top of each other. For metal surfaces such distorted, nearly incommensurate structures commonly occur, ${ }^{19}$ and it would not be surprising if they also occur for a system of (111)-oriented Al planes (2.86 $\AA$ atomic spacing) on AlN (3.11 $\AA$ spacing of $\mathrm{Al}$ atoms). Our model is certainly not intended to provide a full explanation of the observed structure, but rather, it simply provides some specificity in discussing the types of structures which may arise.

In Fig. 6 the solid dots show a hexagonal lattice, and overlaid on that the open dots show a rotated lattice with slightly shorter lattice spacing. In this computation we can use the experimental value of $\vec{k}_{C}$ (or $\vec{k}_{D}$ ) for the overlaid lattice, thus producing an incommensurate fit with the $1 \times 1$ lattice, and the result is very similar to that seen in Fig. 6. 

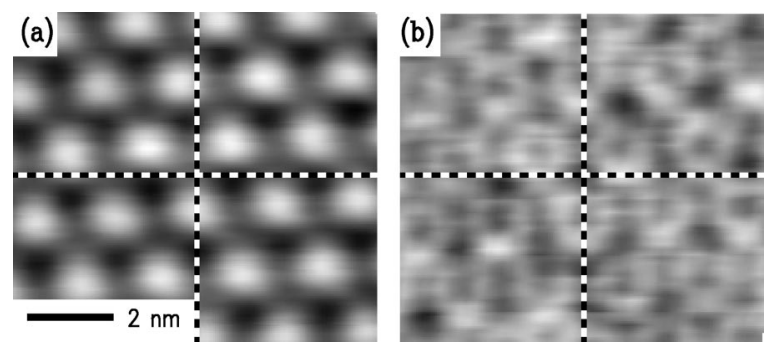

FIG. 7. Voltage dependence of STM images for the $5 \sqrt{3}$ $\times 5 \sqrt{3}-R 30^{\circ}$ reconstruction, (a) $+1.5 \mathrm{~V}$, gray scale of $1.0 \AA$, (b) $-2 \mathrm{~V}$, gray scale of $0.2 \AA$. These images were acquired from the same surface location, with the dashed lines located at identical surface positions.

However, to be specific, we have chosen in Fig. 6 a particular coincidence between the two lattices: Consider a vector of the first $1 \times 1$ lattice of length $5 \sqrt{3} a$ and directed along the $[10 \overline{1} 0]$ direction. In terms of the axes shown in Fig. 6 this vector has an [2 $\overline{1} \overline{1} 0]$ component of $7.5 a$ and a [01 0$]$ component of $2.5 \sqrt{3} a$, and there are 10 lattice planes normal to and intersecting the vector. To form the second lattice, contract and rotate this vector so that its endpoint now has a

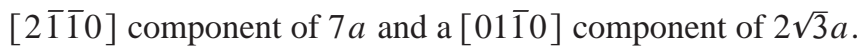
The resulting vector is thus contracted by 0.91085 (corresponding to a primary wave vector of $1.109 \mathrm{~b}$ ) and it is oriented at $3.6705^{\circ}$ relative to $[10 \overline{1} 0]$. The resultant lattice is shown by the open circles in Fig. 6. The coincidence lattice thus formed clearly has hexagonal symmetry, with lattice spacing and orientation in agreement with the STM images.

Regarding the Al coverage of the $2 \sqrt{3} \times 2 \sqrt{3}-R 30^{\circ}$ and $5 \sqrt{3} \times 5 \sqrt{3}-R 30^{\circ}$ surfaces, we return to the AES results of Fig. 1. Two data points for surfaces that display a $2 \times 6$ RHEED pattern are indicated there, revealing a coverage between 2 and $3 \mathrm{ML}$. As noted above, the line intensities for our $2 \times 6$ patterns do vary from surface to surface and across a given surface, and we associate these variations with the presence of a mixture of $2 \sqrt{3} \times 2 \sqrt{3}-R 30^{\circ}$ and $5 \sqrt{3}$ $\times 5 \sqrt{3}-R 30^{\circ}$ phases (with this mixture being seen directly in the STM images). Nevertheless, even with this mixed surface phase, the range of AES ratios for which we obtain a $2 \times 6$ RHEED pattern is quite small, corresponding to about $0.4 \mathrm{ML}$ of Al. With consideration now of the STM images of the mixed surfaces, Figs. 3 and 4, the step heights in these images are all within $0.3 \AA$ of $2.5 \AA$ (one-half the AlN $c$-axis lattice constant), indicating qualitatively that the $\mathrm{Al}$ coverage difference between the $2 \sqrt{3} \times 2 \sqrt{3}-R 30^{\circ}$ and $5 \sqrt{3}$ $\times 5 \sqrt{3}-R 30^{\circ}$ surface should be either $0 \mathrm{ML}$ or a nonzero, integer number of ML's. The former case is compatible with the AES, and we conclude that $\mathrm{Al}$ coverage for the two surfaces is quite close. By a comparison of combined STM, RHEED, and AES results for specific surfaces, we tentatively conclude that the $5 \sqrt{3} \times 5 \sqrt{3}-R 30^{\circ}$ surface has slightly higher Al coverage than the $2 \sqrt{3} \times 2 \sqrt{3}-R 30^{\circ}$.

The voltage dependence of the STM images for the $5 \sqrt{3}$ $\times 5 \sqrt{3}-R 30^{\circ}$ reconstruction is displayed in Fig. 7 . For empty states (corresponding to positive sample voltages) as seen in Figs. 2-4 and 7(a), the images consist of a single corrugation maximum per unit cell with no additional substructure. The filled-state images are much different however, as seen in Fig. 7(b). Now each cell contains a ring-shaped corrugation, with corrugation height an order of magnitude smaller than that for the empty states. The corrugation maxima of the empty states occurs at the center of a filled-state ring. Imaging of both empty and filled states is possible at low voltages with magnitudes on the order of $0.1 \mathrm{~V}$, indicating that the surface has at least some metallic character. On the other hand, the marked voltage dependence displayed in Fig. 7 indicates that the surface is certainly not highly metallic, i.e., with uniform density of states. We conclude that the $5 \sqrt{3}$ $\times 5 \sqrt{3}-R 30^{\circ}$ structure appears to have weakly metallic character, indicative of fractional electron occupation in some bonds.

\section{High Al coverage}

Let us now consider surface structures that form at higher $\mathrm{Al}$ coverages. Such surfaces form naturally following MBE growth if that growth is performed under sufficiently Al-rich conditions. Alternatively, we have formed surfaces with high $\mathrm{Al}$ coverage by deposition of $\mathrm{Al}$, with the sample held at a slightly elevated temperature of $\approx 150^{\circ} \mathrm{C}$, onto the $2 \times 6$ surface discussed in the preceding section or onto a $1 \times 1$-nitrided surface prepared as described in the following section. This deposited $\mathrm{Al}$ produces a flat film, whose thickness increases with the amount of deposited material, as evidenced by an increasing Al/N AES ratio shown in Fig. 1(b) and by STM study of the corresponding surface morphology. Figure 8(a) shows a STM image of a surface that is covered by this type of thick $(\approx 5$-ML) Al layer. High-magnification STM images generally do not reveal any atomic corrugation on the surface, although sometimes a very weak corrugation with an approximate $1 \times$ spacing can be seen, as in Fig. 8(b). In RHEED, this type of surface displays sharp first-order streaks, located at distinctly larger wave vectors than those for a $1 \times 1 \mathrm{AlN}$ surface as illustrated in Fig. 8(c). We refer to this type of RHEED pattern as " $1 \times 1-\mathrm{Al}$," with the observed streaks being located at wave vectors $(6 \pm 1) \%$ larger than that for the AlN $1 \times 1$ surface.

Thermal annealing at temperatures above about $750{ }^{\circ} \mathrm{C}$ for 5-10 min is found to effectively remove the "thick" Al film from the surface. We have examined such films after annealing and we find $\mu \mathrm{m}$-size $\mathrm{Al}$ droplets on the surface, similar to that reported by Koblmueller et al. ${ }^{18}$ The RHEED pattern of Fig. 8(d) was acquired from such an annealed surface, maintained at the elevated temperature. With sufficient annealing time the $\mathrm{Al} / \mathrm{N}$ Auger ratio returns to a value close to that of the $2 \times 6$ structure, and the RHEED pattern displays $2 \times 6$ symmetry, as shown in Fig. $8(\mathrm{e})$. In contrast, Fig. 8(f) shows the pattern obtained from a surface that started with the "thick" Al film and was then annealed for a time insufficient to produce the $2 \times 6$ structure. The result is a pattern that displays streaks at the $1 \times$ positions of $\mathrm{AlN}$ and neighboring streaks located at wave vectors $(10 \pm 1) \%$ larger than the $1 \times$ spacing. The wave vector of these latter streaks is thus intermediate between those of the $1 \times 1-\mathrm{Al}$ pattern [Fig. 8(c)] and those of the $2 \times 6$ pattern [Fig. 8(e) or 5(b)]. 

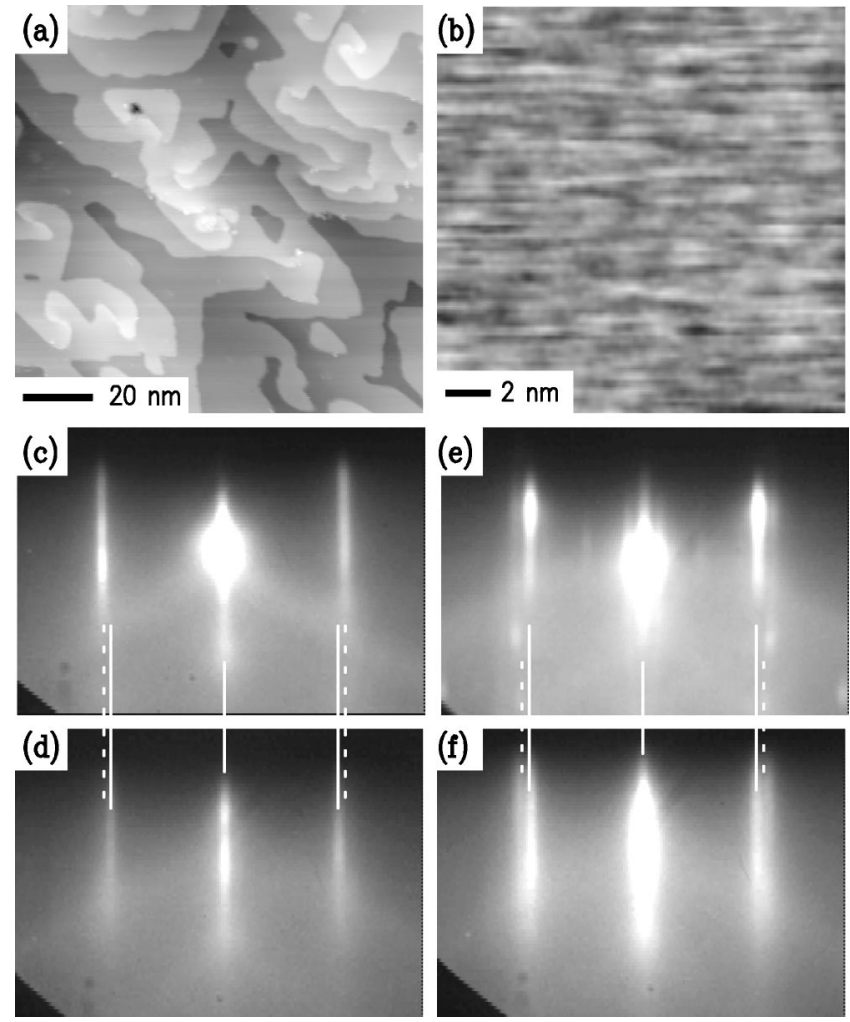

FIG. 8. (a) Large-scale STM image of surface covered with Al film. Sample voltage is $-4 \mathrm{~V}$; gray scale range is $12 \AA$. (b) Higher magnification view of same surface. Sample voltage is $-0.5 \mathrm{~V}$; gray scale range is $0.3 \AA$. (c)-(f) RHEED images with electron beam along $\langle 2 \overline{1} \overline{1} 0\rangle$ : (c) $20^{\circ} \mathrm{C}$, surface covered by a $\approx 5$-ML-thick Al film; (d) $750{ }^{\circ} \mathrm{C}, 15$-min anneal of same surface as in (c); (e) $20^{\circ} \mathrm{C}$, following cool down as same surface as in (d); (f) $20^{\circ} \mathrm{C}$, following cool down of a similar surface as in (d) but that was annealed for only $10 \mathrm{~min}$. White solid lines indicate the location of first-order AlN streaks, and dashed lines indicate first-order streaks associated with the $\mathrm{Al}$ overlayer as in (c).

LEED patterns obtained from surfaces with high $\mathrm{Al}$ coverage are shown in Fig. 9 for two different surfaces. The Al coverages of these surfaces were not well calibrated, but they are known to be in the range of 3-5 ML and with the results from Figs. 9(c) and 9(d) being from a thicker film than that of Figs. 9(a) and 9(b). For the thinner film we note the presence of the spots corresponding to the $2 \sqrt{3} \times 2 \sqrt{3}-R 30^{\circ}$ structure, although these spots have disappeared for the thicker film. In both cases we observed a threefold splitting of the first-order LEED spots, with satellite features $B$ and $C$ having angular orientations of $\vec{k}_{B}-\vec{k}_{A}$ and $\vec{k}_{C}-\vec{k}_{A}$, being at $30 \pm 2^{\circ}$ from a $\langle 01 \overline{1} 0\rangle$ direction. For the $80 \mathrm{eV}$ electron energy used in Fig. 9 spots $A, B$, and $C$ have nearly equal intensity. For lower energy near $70 \mathrm{eV}$ the $B$ and $C$ spots are much more intense than $A$, and for higher energies near 100 $\mathrm{eV}$ the $A$ spot is most intense. The magnitude of $k_{B}$ and $k_{C}$ is found to be $1.106 \pm 0.006$ times $k_{A}$ from Fig. 9(d). Very weak satellite features $D$ and $E$, located along the line joining $B$ and $C$, can also be seen in Figs. 9(c) and 9(d).

Relating the RHEED and LEED results for high $\mathrm{Al}$ coverage is not straightforward. A cursory examination of Figs.
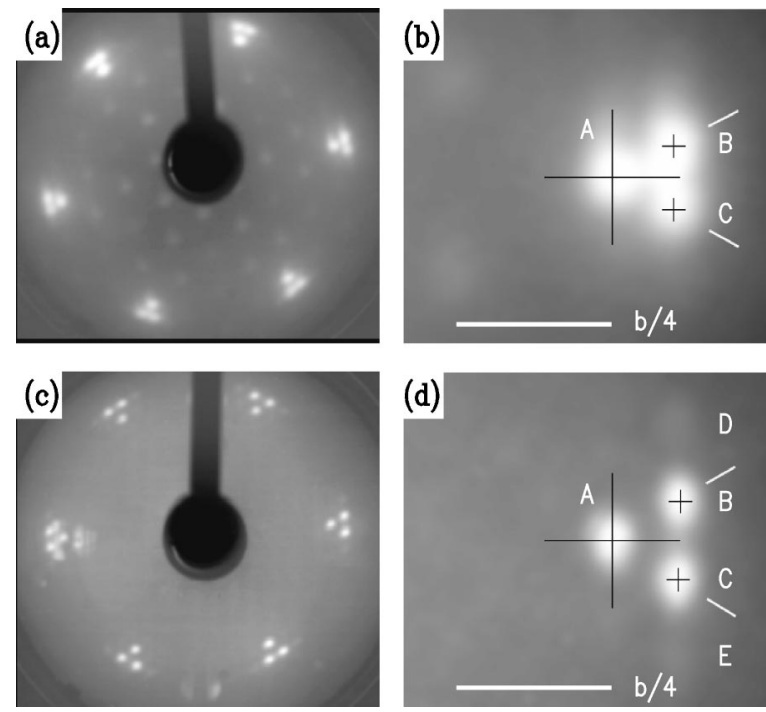

FIG. 9. LEED patterns, acquired at $80 \mathrm{eV}$ electron energy, of $\mathrm{Al}$ films with thickness of a few ML deposited on AlN(0001). Panels (b) and (d) shows expanded views of the first-order reflections and satellite peaks from (a) and (c), respectively. The leftmost first-order peak in (c) is slightly distorted due to a stray light reflection in the optical system used to acquire the image.

8 and 9 suggests agreement of the threefold split LEED patterns with the RHEED results of Fig. 8(f), i.e., obtained from a surface from which some excess $\mathrm{Al}$ had been removed by annealing. However, both the surfaces from which the LEED patterns of Fig. 9 were obtained actually displayed in RHEED a pattern like that of Fig. 8(c), i.e., the $1 \times 1 \mathrm{Al}$ pattern corresponding to high $\mathrm{Al}$ coverage. We have repeatedly observed this type of $1 \times 1 \mathrm{Al}$ RHEED pattern [as in Fig. 8(c)] from surfaces that displayed the threefold splitting of the LEED spots [as in Fig. 9(c)], although it is nontrivial to reconcile the two types of results. Careful measurement of the position of the $A$ spots in the data of Figs. 9(a) and 9(b) reveals that they have wave-vector magnitude within $1 \%$ of the $1 \times$ spacing expected from the $2 \sqrt{3}$ spots seen in the pattern, that is, they cannot have a $(6 \pm 1) \%$ expanded wave vector as might be expected from the RHEED pattern.

A similar statement cannot be made for the data of Figs. 9(c) and 9(d) since the $2 \sqrt{3}$ spots are no longer visible there (and slight experiment-to-experiment variations in the incident electron energy in our LEED system prevents absolute comparison of spot positions between different patterns), but the strong similarity of Figs. 9(a) and 9(c) does suggest that the patterns arise from essentially identical structures. To reconcile the high-Al RHEED and LEED results [Figs. 8(c) and 9(c), respectively], we consider the coherence length of the measurements. Assuming a spread in energy of $0.5 \mathrm{eV}$ for the electron beams in both cases, we estimate coherence lengths of $70 \AA$ for the 80 -eV LEED measurement and 500 $\AA$ for the $15-\mathrm{keV}$ RHEED. ${ }^{20}$ Now, if the surface is composed of small domains of the $\mathrm{Al}$ film that are distorted relative to the AlN $1 \times 1$ lattice and have a typical size of $25 \AA$, then in LEED we will see the superposition of the domains, thus producing the threefold split pattern. However if the domains themselves are incoherent relative to each other (with respect 
to the overlying $\mathrm{Al}$ film), then in RHEED we expect to see the average lattice spacing, thus producing the $1 \times 1 \mathrm{Al}$ pattern. The observed $(6 \pm 1) \%$ increased spacing of the RHEED streaks in Fig. 8(c) would thus be seen to be an average of the positions of the $A, B$, and $C$ spots in the LEED pattern of Fig. 9(c) [a simple average of the $\langle 01 \overline{1} 0\rangle$ components of those wave vectors yields a value (7.0 $\pm 0.6) \%$ greater than the $1 \times$ spacing].

Regarding the origin of the threefold splitting seen in the LEED, we first note that the LEED features are very similar to those seen previously for the soliton-type surface reconstructions of $\mathrm{Au}(111),{ }^{21,22}$ in which the top Au layer is contracted relative to the underlying layers. However, as argued in the prior paragraph, we believe that LEED reveals the structure of not only the surface layer but also subsurface layers. The presence of nearly hexagonal, (111)-oriented Al layers in the film is clear from the diffraction data. From the first-order streak in RHEED there appears to be a uniform $(6 \pm 1) \%$ contraction of this lattice, and the LEED results indicate some additional, more complicated distortions of the thin $\mathrm{Al}$ film. The LEED pattern probably arises from multiple domains of distorted (111) Al layers, and based on the similarity of their respective diffraction patterns we propose that the $5 \sqrt{3} \times 5 \sqrt{3}-R 30^{\circ}$ structure may serve as a template for subsequent growth of the thin $\mathrm{Al}$ film. Certainly a contraction of $\mathrm{Al}(111)$ planes on $\mathrm{AlN}(0001)$ is expected based on their lattice constants, with $\mathrm{Al}-\mathrm{Al}$ spacings of $2.86 \AA$ and $3.11 \AA$, respectively $[-8.0 \%$ change in $\mathrm{Al}-\mathrm{Al}$ spacing of $\mathrm{Al}(111)$ compared to $\mathrm{AlN}]$.

\section{Low Al coverage}

To obtain surfaces that are more N-rich than those displaying a $2 \times 6$ RHEED pattern we use the following procedure: An as-grown surface is heated to $700-750{ }^{\circ} \mathrm{C}$ and exposed to the $\mathrm{N}$ plasma for a period of about $20 \mathrm{~min}$. During this time the $1 \times 1$ RHEED pattern of the surface stays streaky and sharp. The sample is then cooled down under the $\mathrm{N}$ plasma. A brightening of the RHEED pattern is seen when the surface passes through the melting point of $\mathrm{Al}\left(660^{\circ} \mathrm{C}\right)$ and at that point the plasma is turned off (failure to turn off the plasma at that point leads to surface roughening). The resulting RHEED pattern is $1 \times 1$ with sharp streaks, and we refer to it as " $1 \times 1$-nitrided." The $\mathrm{Al}$ content of such a surface can vary considerably, as illustrated by the two different $1 \times 1$-nitrided data points in the AES results of Fig. 1(a). However, a lower limit for the $\mathrm{Al}$ content, i.e., a saturated $\mathrm{N}$ content while still maintaining a flat surface, is observed, as indicated by the lowest data point in Fig. 1(a). In some cases (i.e., for a particular surface coverage of $\mathrm{Al}$ ) this procedure of forming a $1 \times 1$-nitrided surface results in an observed 1 $\times 3$ pattern, shown in Fig. 10, indicative of a $\sqrt{3}$ $\times \sqrt{3}-R 30^{\circ}$ reconstruction. This $\sqrt{3} \times \sqrt{3}-R 30^{\circ}$ surface can also be obtained from a $1 \times 1$-nitrided surface (with saturated $\mathrm{N}$ content) by careful $\mathrm{Al}$ deposition and annealing. In any case, the range of $\mathrm{Al}$ coverages over which the $\sqrt{3}$ $\times \sqrt{3}-R 30^{\circ}$ surface is formed is quite narrow.

One additional $\mathrm{N}$-rich surface reconstruction, namely a $2 \times 2$, has been occasionally observed. We find this structure
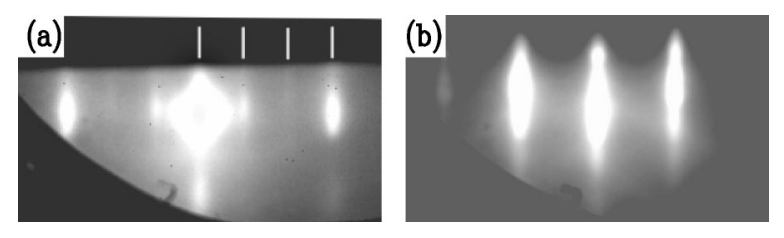

FIG. 10. RHEED patterns displaying $1 \times 3$ pattern, with electron beam along (a) $\langle 01 \overline{1} 0\rangle$ direction and (b) $\langle 2 \overline{1} \overline{1} 0\rangle$ direction. Tic marks in (a) mark the $3 \times$ spacing.

when the MBE growth is performed under Al-poor conditions, i.e., with reduced $\mathrm{Al}$ flux compared to our usual conditions. A sharp, streaky $1 \times 1$ RHEED pattern is still found during growth. If we terminate the growth by closing the $\mathrm{Al}$ shutter, and then cool down the sample under the $\mathrm{N}$ plasma, the $2 \times 2$ pattern forms. We have not studied the $1 \times 1$-nitrided, $2 \times 2$, or $\sqrt{3} \times \sqrt{3}-R 30^{\circ}$ surfaces in any detail by STM, but a few attempts at studying the former surface indicated a disordered surface arrangement.

\section{THEORY: FIRST-PRINCIPLES TOTAL ENERGY CALCULATIONS}

We turn to theory to provide guidance as to energetically stable structures for the reconstructions observed here. Firstprinciples total energy calculations have been performed for a large number of possible AlN(0001) surface reconstructions. The calculations employ the local density functional theory with the Ceperley and Alder exchange-correlation energy functional. The electron-ion interaction is treated using first-principles pseudopotentials. ${ }^{23-26}$ In previous studies of the $A l N(0001)$ surface the guiding principle underlying the choice of structures was satisfaction of the electron-counting rule (ECR), and so the focus was on structures having doubly occupied $\mathrm{N}$ dangling bonds and empty $\mathrm{Al}$ dangling bonds. ${ }^{27}$ This type of semiconducting electronic structure typically necessitates a $2 \times 2$ reconstruction on III-V (0001) surfaces.

In the present work we augment and extend these studies by performing calculations for structures having $\sqrt{3} \times \sqrt{3}$ symmetry and compare the energies of these with the lowest energy $2 \times 2$ structures found previously. As discussed above, a $\sqrt{3} \times \sqrt{3}$ symmetry is observed experimentally in some cases. Moreover, this type of unit cell allows us to consider the laterally contracted metal adlayer structures that have been shown to be stable on $\mathrm{GaN}(0001)$ surfaces under Ga-rich growth conditions. ${ }^{28}$ As discussed below, metallic laterally contracted $\mathrm{Al}$ adlayer structures are found to be energetically favorable in Al-rich conditions in comparison to the standard $2 \times 2$ structures. In this respect AlN and GaN exhibit similar behavior.

The supercell employed in the calculations consists of 4 layers of $\mathrm{Al}, 4$ layers of $\mathrm{N}$, and a layer of pseudohydrogen atoms having charge $3 / 4$ to passivate the back side of the slab. The (0001) surface on the other side of the slab may be decorated by $\mathrm{Al}$ or $\mathrm{N}$ adatoms, or one or two adlayers of $\mathrm{Al}$ may be present. The plane wave cutoff is $50 \mathrm{Ry}$. For the $\sqrt{3} \times \sqrt{3}$ and $2 \times 2$ structures a mesh of 6 special $k$ points is employed to sample the Brillouin zone. The relative energies are calculated as a function of the chemical potential of $\mathrm{Al}$, 


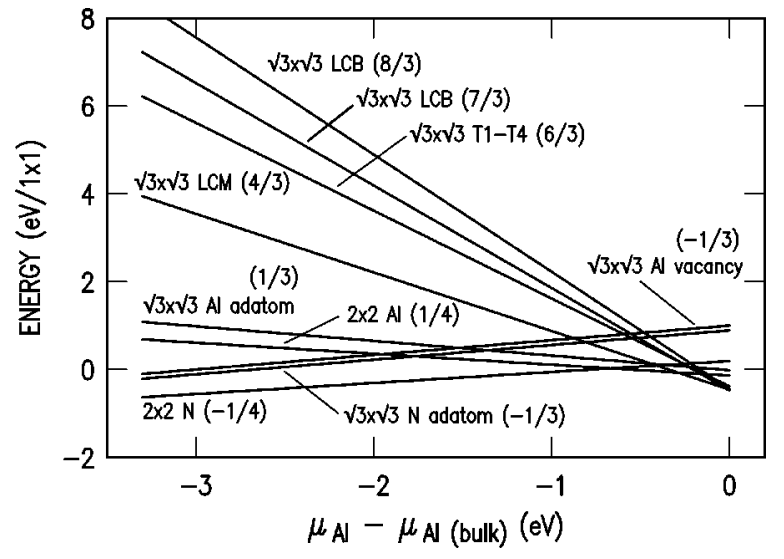

FIG. 11. Relative energies for a variety of $2 \times 2$ and $\sqrt{3} \times \sqrt{3}$ surface reconstructions of the AlN(0001) surface plotted as a function of the chemical potential of Al. The energies are relative to that of the $1 \times 1$ relaxed ideal surface. The zero of the Al chemical potential corresponds to bulk fcc Al. In the N-rich limit, where $\mu_{\mathrm{Al}}$ is lower than the maximum value by $3.3 \mathrm{eV}$, the $2 \times 2 \mathrm{~N}$ adatom model has the lowest surface energy. As the chemical potential is increased the $2 \times 2 \mathrm{Al}$ adatom model becomes lower in energy, and then the $\sqrt{3} \times \sqrt{3}$ LCM structure is preferred. In the Al-rich limit, the $\sqrt{3} \times \sqrt{3}$ LCB structure is preferred by a small amount. The numbers in parentheses refer to the excess (or deficit) in the number of $\mathrm{Al}$ atoms relative to the ideal $1 \times 1$ surface, per $1 \times 1$ unit cell. Many possible models (not shown here) can be excluded on the basis of calculations performed in Ref. 27.

which varies between a lower limit of $\mu_{\mathrm{Al}}=\mu_{\mathrm{Al}(\text { bulk })}-\Delta H$ and an upper limit of $\mu_{\mathrm{Al}}=\mu_{\mathrm{Al}(\mathrm{bulk})}$ as discussed elsewhere. ${ }^{27,29}$ For AlN the formation energy from bulk Al and molecular $\mathrm{N}_{2}$ (at zero temperature) is $\Delta H=3.3 \mathrm{eV} .^{27}$ The calculated energies for a subset of the structures considered in this work are shown in Fig. 11.

Over a large region of the chemical potential space on the $\mathrm{N}$-rich side of the phase diagram the most stable structure that has been reported to date is the $2 \times 2 \mathrm{~N}$ adatom model, in which the adatom occupies an $\mathrm{H}_{3}$ site. ${ }^{27}$ This structure is nonmetallic, with the $\mathrm{N}$ adatom accepting $3 / 4$ of an electron from the $\mathrm{Al}$ rest atom. As the $\mathrm{Al}$ chemical potential is increased, the $2 \times 2 \mathrm{Al} T_{4}$ adatom structure becomes stable in a small region of the chemical potential space. ${ }^{27}$ The $2 \times 2$ structure observed in the present work and in past experiments ${ }^{6,7,9,12}$ could in principle correspond to either of these possibilities.

Adatom structures having $\sqrt{3} \times \sqrt{3}$ symmetry were also considered: As seen in Fig. 11, both the $\mathrm{N}_{3}$ and $\mathrm{Al} T_{4}$ structures are energetically unfavorable for all conditions. Because these structures do not contain rest atoms, the ECR is not satisfied. (The presence of subsurface donors such as oxygen or $\mathrm{Si}$ would be required in each case to satisfy the ECR.) One may conclude from these results that $\sqrt{3} \times \sqrt{3}$ adatom structures would not occur on impurity-free AlN surfaces. The $\sqrt{3} \times \sqrt{3}$ Al vacancy structure and a number of other $\sqrt{3} \times \sqrt{3}$ structures were tested and found to be unstable for all chemical potentials.

As the Al chemical potential is increased, metallic structures having more than one $\mathrm{ML}$ of $\mathrm{Al}$ eventually become
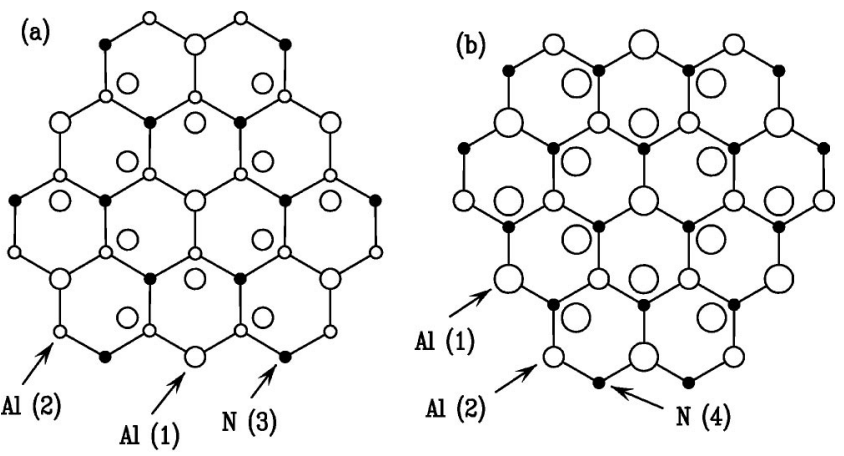

(c)

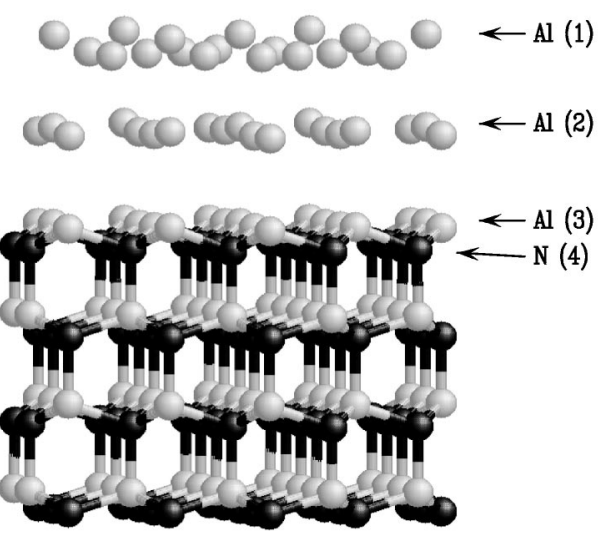

FIG. 12. (a) and (b) Schematic top views of the LCM and LCB models, respectively. Aluminum atoms are shown by open circles and nitrogen atoms by closed circles, with the diameter of the circles representative of the height of the atoms relative to the surface. Layer numbers are given in parentheses. In each case layer 1 is the outermost layer. (b) Three-dimensional rendering of a side view of the LCB model. Aluminum atoms are shown by gray spheres and nitrogen atoms by black spheres. A projection slightly rotated from $\langle 01 \overline{1} 0\rangle$ is chosen, to illustrate the vertical corrugation of the $\mathrm{Al}$ adlayers. In this structure the $\mathrm{Al}$ atoms in layer 2 are positioned directly above those in layer 3 . The $\mathrm{Al}$ atoms in layer 1 that are positioned directly above those in layers 2 and 3 are higher than the other atoms in layer 1 by $\approx 0.5 \AA$.

more stable than the $2 \times 2 \mathrm{Al}$ adatom model. One such model has a $\sqrt{3} \times \sqrt{3}$ symmetry and contains $4 / 3 \mathrm{ML}$ of $\mathrm{Al}$ above the ideal surface. This structure may be described as a laterally contracted monolayer (LCM). It may be viewed as a hexagonal lattice of $\mathrm{Al}$ with lattice vectors reduced by a factor $\sqrt{3} / 2$ and rotated by $30^{\circ}$ so that it is in registry with a $\sqrt{3} \times \sqrt{3}$ cell of the substrate. As shown in Fig. 11 this structure is stable with respect to the $\mathrm{Al} T_{4}$ adatom structure for $\mu_{\mathrm{Al}}$ $>\mu_{\mathrm{Al}(\text { bulk })}-0.29 \mathrm{eV}$. Because of its relative stability in a significant region of the chemical potential space, this structure is a candidate to explain the existence of the $\sqrt{3} \times \sqrt{3}$ structures observed in the present work and in past works. ${ }^{10,11,13}$ A schematic representation of the $\sqrt{3} \times \sqrt{3}$ LCM model is shown in Fig. 12(a).

In the extreme Al-rich limit, where $\mu_{\mathrm{Al}}=\mu_{\mathrm{Al} \text { (bulk) }}$, it is energetically favorable (albeit by a very small amount) to add another layer of $\mathrm{Al}$ to the surface and form a laterally contracted bilayer structure with $7 / 3 \mathrm{ML}$ of excess Al. The structure is shown in Figs. 12(b) and 12(c). In each $\sqrt{3} \times \sqrt{3}$ cell there are 4 atoms in the laterally contracted top layer, 
and 3 atoms in $T_{1}$ sites in the layer underneath. In the registry having the lowest energy, one of the atoms in the top layer is directly above an $\mathrm{Al}$ atom in the $T_{1}$ layer below, and the other three are in bridge sites. The top layer then exhibits substantial corrugation: The atoms above the $T_{1}$ sites reside $\approx 0.53 \AA$ above the other three atoms in the top layer.

It is clear that $1 \times 1 \mathrm{Al}$ adlayer structures having 1 or 2 $\mathrm{ML}$ of $\mathrm{Al}$ are under tensile stress because the $\mathrm{Al}-\mathrm{Al}$ spacing in the adlayer is too large. Two specific examples illustrate this point: Compared to a $1 \times 1$ adlayer structure with $1 \mathrm{ML}$ of $\mathrm{Al}$ in $T_{1}$ sites, the $\mathrm{Al}-\mathrm{Al}$ spacing in the 4/3-ML laterally contracted monolayer structure is reduced by $\approx 13 \%$ and the energy is lower by $0.59 \mathrm{eV} /(\sqrt{3} \times \sqrt{3}$ cell $)$ in the Al-rich limit. A similar result is found in comparing structures with two adlayers. The 7/3-ML laterally contracted bilayer structure is more stable than a 2-ML structure, with adlayers in $T_{1}$ and $T_{4}$ registry, by $0.27 \mathrm{eV} /(\sqrt{3} \times \sqrt{3}$ cell $)$ in the Al-rich limit. Additional confirmation of the existence of tensile stress is obtained by performing calculations for freestanding hexagonal layers of $\mathrm{Al}$ as a function of the lattice constant. The equilibrium lattice constant found in such calculations is $\approx 2.71 \AA$. This is substantially less than the equilibrium inplane lattice constant of AlN. These results, together with the finding that considerable vertical corrugation occurs in the laterally contracted bilayer structure, support the view that the surfaces seen in the STM images under Al-rich conditions result from an optimization of stress relief in $\mathrm{Al}$ adlayers.

In summary, the theoretical results demonstrate that the $2 \times 2 \mathrm{~N} \mathrm{H}_{3}$ adatom model is relatively stable over a substantial region of the chemical potential space, and is therefore a plausible model to explain the $2 \times 2$ structure seen in N-rich conditions. Based on the calculations the most likely model to explain a $\sqrt{3} \times \sqrt{3}$ symmetry structure is the $4 / 3$-ML laterally contracted monolayer structure depicted in Fig. 12(a). In very Al-rich conditions the calculations indicate that metallic adlayer structures having more than one $\mathrm{ML}$ of excess $\mathrm{Al}$ are energetically favorable. These adlayers are stabilized by a contraction of the Al-Al spacing to relieve tensile stress. Experimentally we observe a $2 \sqrt{3} \times 2 \sqrt{3}-R 30^{\circ}$ structure at this coverage. Perhaps this type of structure can be formed from the 7/3-ML structure by appropriate buckling of the surface atoms, or alternatively a more complex rearrangement is needed. In any case a $30^{\circ}$-rotated arrangement of either the first or second layers (or both) seems likely to occur.

Considering now the $5 \sqrt{3} \times 5 \sqrt{3}-R 30^{\circ}$ structure, observed at Al coverage close to that of the $2 \sqrt{3} \times 2 \sqrt{3}-R 30^{\circ}$ structure, we propose that this structure arises from $\mathrm{a} \approx 4^{\circ}$ rotation and $\approx 9 \%$ contraction of the surface lattice of $\mathrm{Al}$ as described qualitatively in Sec. III B, which produces a surface Al-Al separation close to that of unstrained $\mathrm{Al}(111)$ planes. The unit cell of the resulting structure is much too large for the first-principles theory to handle. Future work using more approximate potentials is needed to test such models, and in particular to compare their energy relative to the $7 / 3-\mathrm{ML}$ structure. Finally, at higher $\mathrm{Al}$ coverages we observe the formation of $\mathrm{Al}$ layers with a contracted lattice compared to the AlN. The nature of the distortion of this lattice appears to follow that of the $5 \sqrt{3} \times 5 \sqrt{3}-R 30^{\circ}$ structure, and again the net result is that the Al-Al separation in each plane of the film is close to that of bulk Al.

\section{SUMMARY}

The sequence of reconstructions observed in our work is summarized as follows: For Al-poor surfaces we find $2 \times 2$ and $1 \times 3$ RHEED patterns, the latter which we associate with a $\sqrt{3} \times \sqrt{3}-R 30^{\circ}$ reconstruction. For moderately Al-rich surfaces, we observe $2 \sqrt{3} \times 2 \sqrt{3}-R 30^{\circ}$ and $5 \sqrt{3} \times 5 \sqrt{3}-R 30^{\circ}$ reconstructions. The former produces a $2 \times 6$ RHEED pattern, and the latter then adds some satellite features and complex intensity variations (from diffraction spot splittings) in that pattern. For greater Al surface coverage, flat films of epitaxial $\mathrm{Al}$ are found to form on the surface, with a characteristic $1 \times 1 \mathrm{Al}$ RHEED pattern having fringe spacing slightly expanded relative to the $1 \times 1$ AlN spacing and a LEED pattern with a threefold spot pattern.

The sequence of reconstructions observed here is similar to that previously observed by Lebedev et al. for Al-polar AlN(0001) surfaces. ${ }^{11}$ We expect the same polarity in our case since our growths are performed on Ga-polar GaN. (In a few cases we have also overgrown our AlN films with GaN and observed the characteristic reconstruction associated with Ga polarity on those surfaces, ${ }^{4}$ thus further confirming the AlN film polarity.) One notable difference between our results and those of Lebedev et al. ${ }^{11}$ is their observation of a $2 \times 6$ RHEED pattern during growth, at a temperature of $850^{\circ} \mathrm{C}$, whereas we have only observed this pattern after cooling our sample following the growth. We believe that this difference arises from the use, in our case, of more Alrich conditions and somewhat lower growth temperatures such that excess Al (over that required for a the $2 \sqrt{3}$ $\times 2 \sqrt{3}-R 30^{\circ}$ structure) exists on the surface and inhibits the formation of the $2 \sqrt{3} \times 2 \sqrt{3}-R 30^{\circ}$ reconstruction. Following growth at our usual temperatures of $750-800{ }^{\circ} \mathrm{C}$ our surfaces generally display the $1 \times 1 \mathrm{Al}$ RHEED pattern, indicating more than $3 \mathrm{ML}$ of $\mathrm{Al}$ accumulation on the surface; in this respect the higher growth temperatures used in Refs. 11 and 18 may be preferable for PAMBE in order to avoid an overabundance of surface $\mathrm{Al}$ that may inhibit $\mathrm{N}$ incorporation into the growing AlN film.

\section{ACKNOWLEDGMENTS}

The authors thank Dr. S. J. Son for providing high quality MOCVD-grown GaN on sapphire wafers. We also gratefully acknowledge $\mathrm{H}$. McKay and S. Nie for their contributions to the work. This work has been supported in part by the Office of Naval Research under Grants No. N00014-02-1-0933 and No. N00014-02-0433. J.N. thanks the Deutsche Forschungsgemeinschaft for financial support. 
${ }^{1}$ J. Neugebauer, T. K. Zywietz, M. Scheffler, J. E. Northrup, H. Chen, and R. M. Feenstra, Phys. Rev. Lett. 90, 056101 (2003).

${ }^{2}$ R. M. Feenstra, J. E. Northrup, and J. Neugebauer, MRS Internet J. Nitride Semicond. Res. 7, 3 (2002).

${ }^{3}$ A. R. Smith, R. M. Feenstra, D. W. Greve, J. Neugebauer, and J. E. Northrup, Phys. Rev. Lett. 79, 3934 (1997).

${ }^{4}$ A. R. Smith, R. M. Feenstra, D. W. Greve, M.-S. Shin, M. Skowronski, J. Neugebauer, and J. E. Northrup, J. Vac. Sci. Technol. B 16, 2242 (1998).

${ }^{5}$ A. R. Smith, R. M. Feenstra, D. W. Greve, M.-S. Shin, M. Skowronski, J. Neugebauer, and J. E. Northrup, Surf. Sci. 423, 70 (1999).

${ }^{6}$ M. A. L. Johnson, S. Fujita, W. H. Rowland, K. A. Bowers, W. C. Hughes, Y. W. He, N. A. El-Masry, J. W. Cook, J. F. Schetzina, J. Ren, and J. A. Edmond, J. Vac. Sci. Technol. B 14, 2349 (1996).

${ }^{7}$ E. S. Hellman, D. N. E. Buchanan, and C. H. Chen, MRS Internet J. Nitride Semicond. Res. 3, 43 (1998).

${ }^{8}$ A. Bourret, A. Barski, J. L. Rouvière, G. Renaud, and A. Barbier, J. Appl. Phys. 83, 2003 (1998).

${ }^{9}$ S. W. King, C. Ronning, R. F. Davis, M. C. Benjamin, and R. J. Nemanich, J. Appl. Phys. 84, 2086 (1998).

${ }^{10}$ H. P. D. Schenk, G. D. Kipshidze, U. Kaiser, A. Fissel, J. Kräusslich, J. Schulze, and W. Richter, J. Cryst. Growth 200, 45 (1999).

${ }^{11}$ V. Lebedev, B. Schröter, G. Kipshidze, and W. Richter, J. Cryst. Growth 207, 266 (1999).

${ }^{12}$ C. S. Davis, S. V. Novikov, T. S. Cheng, R. P. Campion, and C. T. Foxon, J. Cryst. Growth 226, 203 (2001).

${ }^{13}$ N. Onojima, J. Suda, and H. Matsunami, Appl. Phys. Lett. 80, 76 (2002); J. Cryst. Growth 237-239, 1012 (2002).

${ }^{14}$ We follow the usual notation in quoting RHEED patterns from a surface with hexagonal symmetry, namely, listing the number of fractional-order streaks (plus one for the integer-order streak) observed with the electron beam along $\langle 2 \overline{1} \overline{1} 0\rangle$ and $\langle 01 \overline{1} 0\rangle$ directions, respectively. The latter is not a primitive lattice vector so that, e.g., a $\sqrt{3} \times \sqrt{3}-R 30^{\circ}$ reconstruction leads to a $1 \times 3$ RHEED pattern.

${ }^{15}$ L. E. Davis, N. C. MacDonald, P. W. Palmberg, G. E. Riach, and R. E. Weber, Handbook of Auger Electron Spectroscopy, 2nd ed. (Perkin-Elmer Corporation, Eden Prairie, MN, 1978), p. 13.

${ }^{16}$ S. Mroczkowski and D. Lichtman, Surf. Sci. 131, 159 (1983).

${ }^{17}$ E. J. Tarsa, B. Heying, X. H. Wu, P. Fini, S. P. DenBaars, and J. S. Speck, J. Appl. Phys. 82, 5472 (1997).

${ }^{18}$ G. Koblmueller, R. Averbeck, L. Geelhaar, H. Riechert, W. Hösler, and P. Pongratz, J. Appl. Phys. 93, 9591 (2003).

${ }^{19}$ See, e.g., K. Reshöft, C. Jensen, and U. Köhler, Surf. Sci. 421, 320 (1999).

${ }^{20}$ H. Lüth, Surfaces and Interfaces of Solid Materials, 3rd ed. (Springer, Heidelberg, 1995), pp. 208-209. We use beam divergences of $10^{-2}$ rad for LEED and $10^{-4}$ rad for RHEED, consistent with the specifications of our instruments.

${ }^{21}$ Y. Tanishio, H. Kanamori, K. Takayanagi, K. Yagi, and G. Honjo, Surf. Sci. 111, 395 (1981).

${ }^{22}$ U. Harten, A. M. Lahee, J. P. Toennies, and Ch. Wöll, Phys. Rev. Lett. 54, 2619 (1985).

${ }^{23}$ W. Kohn and L. J. Sham, Phys. Rev. 140, 1133 (1965).

${ }^{24}$ R. Stumpf and M. Scheffler, Comput. Phys. Commun. 79, 447 (1994).

${ }^{25}$ N. Troullier and J. L. Martins, Phys. Rev. B 43, 1993 (1991).

${ }^{26}$ D. M. Ceperley and B. J. Alder, Phys. Rev. Lett. 45, 566 (1980); J. P. Perdew and A. Zunger, Phys. Rev. B 23, 5048 (1981).

${ }^{27}$ J. E. Northrup, R. Di Felice, and J. Neugebauer, Phys. Rev. B 55, 13878 (1997).

${ }^{28}$ J. E. Northrup, J. Neugebauer, R. M. Feenstra, and A. R. Smith, Phys. Rev. B 61, 9932 (2000).

${ }^{29}$ Guo-Xin Qian, R. M. Martin, and D. J. Chadi, Phys. Rev. B 38, 7649 (1988). 